\title{
The Gas Bubble Behavior under Surface Tension Change in Microgravity Conditions
}

\author{
Michael Shoikhedbrod ${ }^{1,2 *}$ \\ ${ }^{1}$ Electromagnetic Impulse Inc. Ontario, Toronto M3J 1K7, Canada \\ ${ }^{2}$ Electromagnetic Impulse Inc., 21 Four Winds Drive, Unit 12, North York, Ontario, Canada
}

\begin{abstract}
The paper presents the study of the influence of surface tension and cohesive angle changes on the behavior of bubbles (drops), fixed on the solid surface, in decreased gravity and microgravity conditions. A new mathematical model of the behavior of the gas bubbles (drops), fixed on the solid surface under the surface tension and cohesive angle changes in decreased and microgravity and has been developed. The developed model permitted to numerically calculate the gas bubbles (drops) profiles behavior and their common surface energy change under surface tension and cohesive angle changes in decreased gravity and microgravity conditions. The developed model permitted also to estimate conditions of bubbles (drops) tear from and fixation on the solid surface under surface tension and cohesive angle change in these conditions. These calculations and estimations have been proved on the conducted parabolic aircraft's tests. The tests showed that the developed model can correctly simulate the gas bubbles and drops profiles behavior, precisely calculate their common surface energy change and estimate conditions of their tear from and fixation on the solid surface in reduced and microgravity conditions.
\end{abstract}

Keywords: Gas bubble; Microgravity; Surface tension; Surface energy; Kinetic energy

\section{Introduction}

Bashforth and Adams [1] first calculated the precise tables of the profiles of the drops of fluids and surface tension. Padday [2] presented the extended tables of the computer calculations of the profiles of the drops of fluids. The published tables can be used only for narrow diapason of drops and do not have universal nature.

In refs. [3,4] first was presented the computer model, which permits to precisely calculate the profiles of any drops of fluids (gas bubbles), to determine their common surface energy and the resultant force, which retains them on the solid surface.

The consideration of the gas bubbles (drops), fixed on the solid surface, behavior under the surface tension and cohesive angle change in decreased gravity and microgravity conditions plays important role in the systems of power supply and life support of the space apparatus.

In this research is presented the theoretical and experimental study of gas bubbles (drops) behavior (positive Bond Number), fixed on the solid surface in a fluid, under the surface tension and cohesive angle changes in decreased gravity and microgravity conditions.

\section{Objectives}

The main objective of the present research is the determination of the influence of surface tension and cohesive angle changes on the single bubble (drop), fixed on a solid surface in a fluid, behavior under decreased gravity and microgravity conditions. For achievement of this objective the following specific tasks were determined:

The calculation of the gas bubble (drop) profiles change under decreased surface tension and increased cohesive angle, under increased surface tension and decreased cohesive angle in decreased gravity and microgravity conditions.

The calculation the gas bubble (drop) common surface energy under decreased surface tension and increased cohesive angle, under increased surface tension and decreased cohesive angle in decreased gravity and microgravity conditions.
The determination of the conditions of bubbles (drops) tear from and fixation on the solid surface (gas bubbles resultant force change) under decreased gravity and microgravity conditions.

\section{Organization of Paper}

Section 2 describes the theoretical study of the gas bubbles (drops) behavior, fixed on a solid surface in the fluid, under surface tension and cohesive angle changes in decreased gravity and microgravity conditions;

Section 3 discusses the results of experimental study of the gas bubbles (drops) behavior, fixed on a solid surface in the fluid, under surface tension and cohesive angle changes in decreased gravity and microgravity conditions.

Fluid under surface tension and cohesive contact angle changes in decreased gravity and microgravity conditions

The developed mathematical model of gas bubble (drop) with positive Bond Numbers behavior fixed on the solid surface in a fluid under decreased, increased gravity, microgravity ("self-gravity") conditions [1] is based on the Laplace condition which is written for common drop (bubble) profile coordinates as:

$$
1 / \mathrm{p}_{\mathrm{i}}+\operatorname{Sin} \mathrm{F} / \mathrm{X}=2+\mathrm{BZ}
$$

and boundary's equation:

$$
2 f f a 6 \operatorname{Sin} F+p g V o l=z 6 a^{2}\left(1 / p_{]}+\operatorname{Sin} F / X\right) \text {. }
$$

*Corresponding author: Shoikhedbrod M, Active Director, Electromagnetic Impulse Inc., 21 Four Winds Drive Unit 12, North York, Ontario, Canada, Tel: 4166509065; Fax: 4166509065; E-mail: mshoikh@primus.ca

Received July 10, 2017; Accepted August 15, 2017; Published August 22, 2017 Citation: Shoikhedbrod M (2017) The Gas Bubble Behavior under Surface Tension Change in Microgravity Conditions. Fluid Mech Open Acc 4: 171. doi: 10.4172/2476-2296.1000171

Copyright: (c) 2017 Shoikhedbrod M. This is an open-access article distributed under the terms of the Creative Commons Attribution License, which permits unrestricted use, distribution, and reproduction in any medium, provided the original author and source are credited. 
Where $\mathrm{p}$ : density equals $\mathrm{p}$ (fluid) for gas bubble case and $\mathrm{p}$ (drop fluid) $-\mathrm{p}$ (fluid) for drop case; $\mathrm{ft}=\mathrm{pgb}^{2} / \mathrm{a}$ : Bond Number; $\mathrm{p}, \mathrm{F}, \mathrm{X}$ and $\mathrm{Z}$ : common main radius and coordinates of bubble's (drop's) profile; Vol: bubble's (drop's) volume; g: Earth gravity, b: multiply coefficient; $\mathrm{a}-=3.1416$, a: surface tension and a: bubble's (drop's) contact radius.

The main principles of the model are:

1. The behavior of the gas bubble (drop), fixed on the surface of solid material is examined in the isothermal, isobaric conditions.

2. Fluid is considered incompressible, i.e. $\mathrm{dp} / \mathrm{dt}=0$ and, therefore, according to the law of mass conservation, volume of gas bubble it is constant.

3. The behavior of gas bubble, which is under the action only of forces of gravity and of surface tension, is examined: external forces are absent.

4. The changes of the surface tension on the border of the section of the phase's fluid-gas and the accelerations of gravity lead simultaneously to a change of the form of gas bubble and its contact wetting angle.

In the present model the influence of changes of the acceleration of gravity and surface tension on the border of the section of the phase's fluid-gas on the form of gas bubble is considered as follows.

With a change of the acceleration of gravity occurs a change of the parameter $\mathrm{II}=\mathrm{pgb}^{2} / 6$, by the changed value of which, the value of one of the coordinates ( $\mathrm{X}$ or $\mathrm{Z}$ ) of the profile of the form of the gas bubble, which corresponds to the new value of acceleration $g$, is determined. Further, in accordance with the law of mass conservation, it is produced the calculation of another coordinate of the profile of the form of gas bubble, thus, that the value of the volumes of bubble, which correspond to the previous value of acceleration $g$ and new, coincided.

After this, it is changed the surface tension on the border of the section of the phase's fluid-gas. In this case, it is changed the parameter/3, by changed value of which, is determined the value of one of the coordinates of the profile of the form of bubble, which corresponds to the new value of a. In accordance with the law of mass conservation, it is produced the calculation of another coordinate of the profile of the form of bubble, thus, that the values of the volumes of bubble, which correspond to the previous values of the acceleration of gravity and of surface tension of 6 and to the new value of a, coincided. The influence of a change of the surface tension on the border of the section of the phase's fluid-gas on the value of the cohesive contact angle of bubble is determined by the formula:

$\operatorname{SinF=-pgVolThraer+a/2}\left(1 / \mathrm{p}_{\mathrm{i}}+1 / \mathrm{p}_{2}\right)$

Where $\mathrm{p}$ : density equals $\mathrm{p}$ (fluid) for gas bubbles case and $\mathrm{p}$ (drop fluid): $p$ (fluid) for drop case; pi, p2, F: common main radiuses of bubble's (drop's) profile, contact wetting angle; Vol: bubble's (drop's) volume; a: -3.1416 , g: Earth gravity, a: surface tension and a: bubble's (drop's) contact radius.

The proposed model permits to numerically calculate the resultant force, which retains the bubble on the surface of solid material and common surface energy change on each step of changes of the surface tension on the border of the section of the phases fluid-gas and cohesive contact angle in the conditions of variable acceleration of gravity by formulas:

$$
\begin{aligned}
& F_{r}=2 f f a 6 \operatorname{SinF}+\text { pgVol }-f f e r a^{2}(1 / p i+1 / p 2) \\
& d E=a l d S 1+d S 2(\operatorname{Cos} F)
\end{aligned}
$$

Here $\mathrm{F}_{\mathrm{r}}$ : resultant force, which retains bubble on the surface of solid material, dE: common surface energy change; dc1, dS2: bubble's (drop's) side surface square change and bubble's (drop's) contact square change, calculated in accordance to the bubble's (drop's) profiles, F: cohesive contact angle. The bubbles (drops) profiles were calculated by Adams method.

The proposed model permits to numerically calculate of the form of gas bubble and, therefore, to estimate the resultant force, which retains it on the surface of solid material and by common surface energy change, using a change of the surface tension on the border of the section of the phases fluid-gas in the conditions of small gravity and weightlessness.

\section{Numerical analysis}

The numerical modeling of gas bubble (drop) with positive Bond Number behavior fixed on the solid surface in a fluid under decreased gravity and microgravity ("self-gravity") conditions was conducted for example with the gas bubbles of volumes of $2.05 \mathrm{~cm}^{3}$. The parameters of gas bubble and fluid were: $\mathrm{p}$ (water) $=1 \mathrm{~g} / \mathrm{cm}^{3}$; a had the following values 55 dyne $/ \mathrm{cm}, 70$ dyne $/ \mathrm{cm}$ and 85 dyne $/ \mathrm{cm}$; in accordance to (3) the cohesive contact angle (F) correspondently had the following values 1.8478 radians, 1.4287 radians and 1.1574 radians. Gravity (g) was changed from $980 \mathrm{~cm} / \mathrm{sec}^{2}$ to $9.8 \mathrm{~cm} / \mathrm{sec}^{2}$ (from 1 to $0.01 \mathrm{~g}$ ).

The carried out numerical calculations of model on the computer showed that for the bubbles with $/ 3>0$, laws governing the behavior of their form with a change of the surface tension on the border of the section of the phases fluid-gas and of cohesive contact angle in the conditions of small gravity, include the following: with an increase of a occurs an increase the height of bubble and the decrease of the perimeter of its fastening on the surface of solid material.

Figure 1a demonstrates the photo stills of the work of the developed computer program, which simulates the profiles of the forms of gas bubble, with a volume of $2.05 \mathrm{~cm}^{3}$, acquired by it with the decrease of
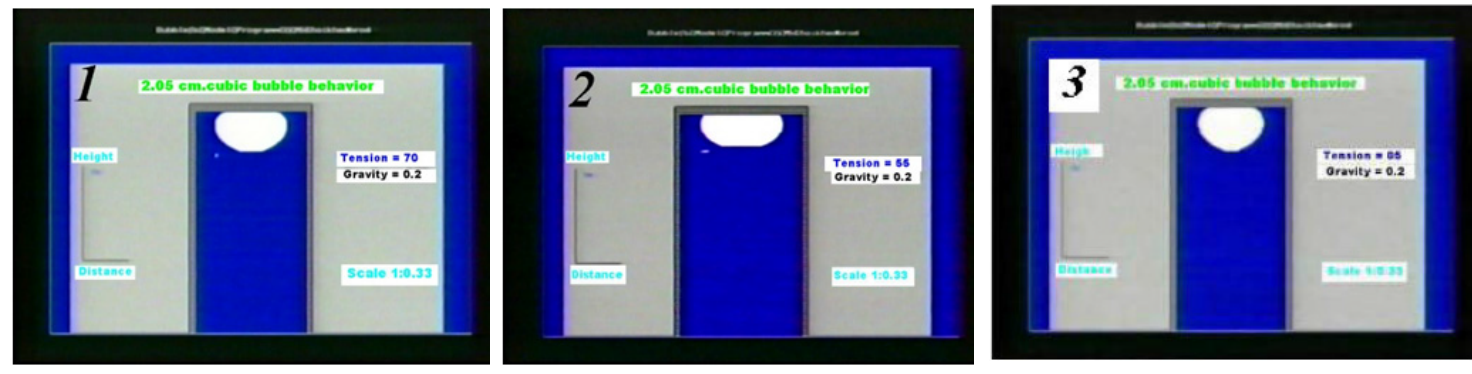

Figure 1a: Stills simulate the profiles of the forms of gas bubble under decrease and increase of a in the conditions of micro-gravity. 

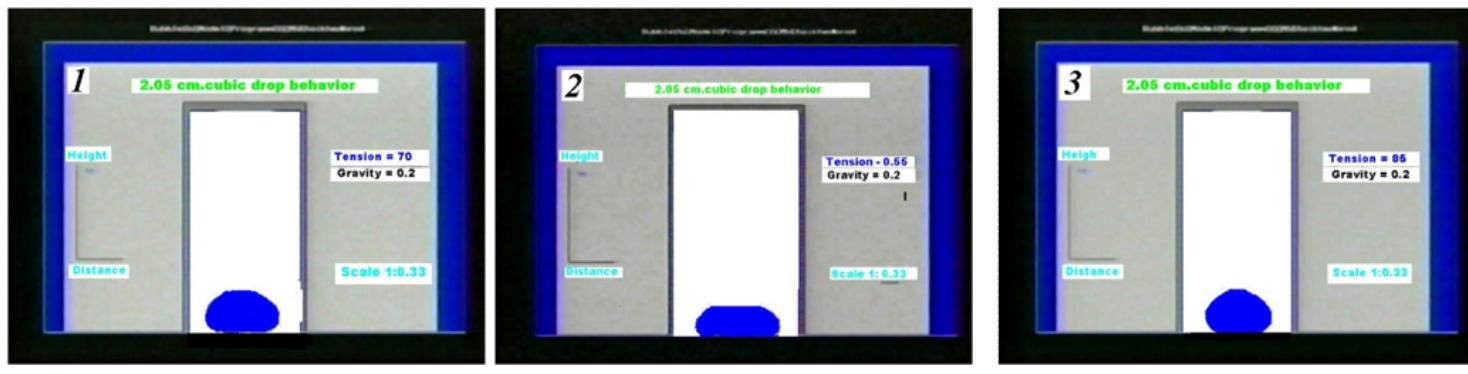

Figure 1b: Stills simulate the profiles of the forms of the drop of water under decrease and increase of a in microgravity.

a from 70 dyne/cm (1- $0=1.4287$ radians) to 55 dyne/cm $(2-0=1.8478$ radians) and an increase of a from 70 dyne $/ \mathrm{cm}$ ( $1-0=1.4287$ radians) to $85 \mathrm{dyne} / \mathrm{cm}(3-0=1.1574$ radians $)$ in the conditions of $\mathrm{g}=196 \mathrm{~cm} / \mathrm{sec}^{2}$ (or $\mathrm{n}=0.2$ un).

Figure $1 \mathrm{~b}$ demonstrates the photo stills of the work of the developed computer program, which simulates the profiles of the forms of the drop of water, with a volume of $2.05 \mathrm{~cm}^{3}$, acquired by it with the decrease of a from 70 dyne $/ \mathrm{cm}$ (1) to 55 dyne/cm (2) and an increase of $\mathrm{r}$ from 70 dyne/cm (1) to 85 dyne/cm (3) in the conditions of $\mathrm{g}=196$ $\mathrm{cm} / \sec ^{2}$ (or $\mathrm{n}=0.2 \mathrm{un}$ ).

Calculations have shown that with an increase of the coefficient of the surface tension of 6 , in the conditions of small gravity occurs the additional release of the surface energy of bubble. 319.2 Erg to 515.51 Erg with a change of the coefficient of the surface tension of a from $\mathrm{a}=70$ dyne $/ \mathrm{cm}$ to and $\mathrm{a}=85 \mathrm{dyne} / \mathrm{cm}$ in the conditions of $\mathrm{g}=196 \mathrm{~cm} / \mathrm{sec}^{2}$.

Thus, using an increase of the coefficient of the surface tension of a (the action of apolar reagent on the complex the bubble-surface of solid material) permitted to control a quantity of the freed energy of gas bubble, which is formed with the decrease of the acceleration of gravity.

Calculations have shown that with decrease of the coefficient of the surface tension of a, in the conditions of small gravity occurs the release of the surface energy of bubble, but less the in case $a=70 \mathrm{dyne} / \mathrm{cm}$.

The results have shown the decrease of the common surface energy of gas bubble from 319.2 Erg to $102.87 \mathrm{Erg}$ with a change of the coefficient of the surface tension of a from $\mathrm{a}=70$ dyne $/ \mathrm{cm}$ to and $\mathrm{a}=55$ dyne $/ \mathrm{cm}$ in the conditions of $\mathrm{g}=196 \mathrm{~cm} / \mathrm{sec}^{2}$.

Carried out numerical calculations of the resultant force, which retains bubble on the surface of solid material, permitted to conclude, that with an increase in the surface tension on the border of the section of the phases fluid-gas in the conditions of small gravity, the resultant force decreases and, therefore, the contact of the bubble-surface of solid material deteriorates.

The results of the calculations have shown that resultant force decrease from 300.23 dynes to 157 dynes with an increase of the coefficient of the surface tension a from $\mathrm{a}=70 \mathrm{dyne} / \mathrm{cm}$ to $\mathrm{a}=85 \mathrm{dyne} / \mathrm{cm}$.

With the decrease of a, in the conditions of small gravity, the resultant force, which retains bubble on the surface of solid material, increases and, therefore, contact of the bubble-surface of solid material is strengthened.

The results of the calculations have shown the increase of resultant force from 300.23 dynes to 452 dynes with an increase of the coefficient of the surface tension from 70 dyne $/ \mathrm{cm}$ to $85 \mathrm{dyne} / \mathrm{cm}$.

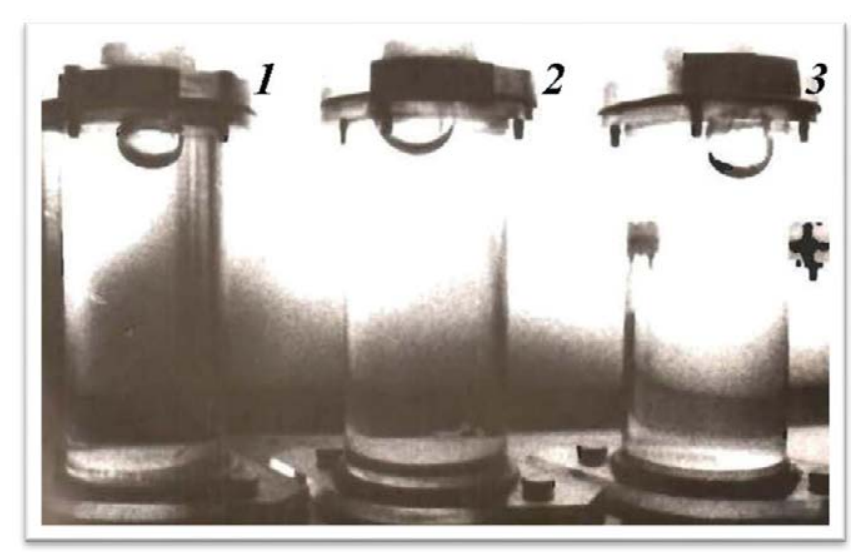

Figure 2: Still of the carried out aircraft's tests shows the profiles of the forms of gas bubble under decrease and increase of 6 in the conditions of $g=196$ $\mathrm{cm} / \mathrm{sec}^{2}$.

Experimental study of the gas bubbles (drops) behavior fixed on a solid surface in a fluid under surface tension and cohesive contact angle changes in decreased gravity and microgravity conditions

The theoretical conclusions and numerical calculations of the developed model gas bubble (drop) with positive Bond Number behavior on the solid surface in a fluid under decreased gravity, microgravity ("self-gravity") conditions have been proved during a parabolic aircraft's tests [5].

The experiments were conducted to gas bubbles with volume of gas bubbles $2.05 \mathrm{~cm}^{3}$ with parameters of gas bubble and fluid: $\mathrm{p}$ (water) $=1$ $\mathrm{g} / \mathrm{cm}^{3}, \mathrm{a}=55,70,85$ dyne $/ \mathrm{cm}$, cohesive contact angle corresponding $\mathrm{F}=1.8478,1.4287,1.1574$ radians. Gravity (g) was changed from 980 $\mathrm{cm} / \mathrm{sec}^{2}$ to $9.8 \mathrm{~cm} / \mathrm{sec}^{2}$ (from 1 to $0.01 \mathrm{~g}$ ).

In Figure 2, it can been seen the still of the filming, obtained during the process of the carried out aircraft's tests, of the profiles of the forms of gas bubble, with a volume of $2.05 \mathrm{~cm}^{3}$, acquired by it with the decrease of a from 70 dyne $/ \mathrm{cm}$ to 55 dyne $/ \mathrm{cm}$ and an increase of a from 70 dyne $/ \mathrm{cm}$ to $85 \mathrm{dyne} / \mathrm{cm}$, in the conditions of $\mathrm{g}=196 \mathrm{~cm} / \mathrm{sec}^{2}$ (or $\mathrm{n}=0.2 \mathrm{un}$ ) that it is completely confirmed with (Figure 1a) of the Section 1.

In Figure 3 it can been seen the still of filming, obtained during of aircraft's tests, of the detachment and forward motion the bubbles with the values of a equal to respectively $70 \mathrm{dyne} / \mathrm{cm}$ and $85 \mathrm{dyne} / \mathrm{cm}$, and the confinement on the surface of the solid material of bubble with $\mathrm{a}=55 \mathrm{dyne} / \mathrm{cm}$ in the short-term conditions of the weightlessness that it is also confirmed with early made theoretical conclusions in the Section 1. 


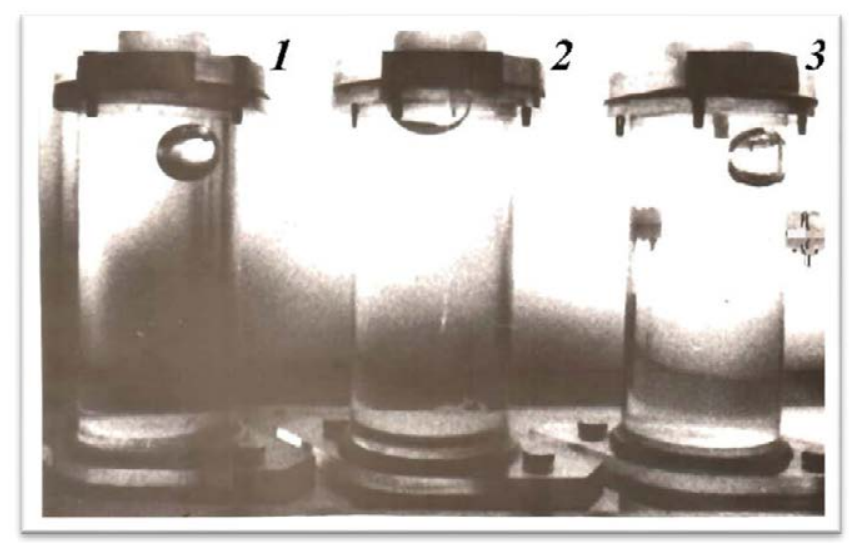

Figure 3: Still of aircraft's tests shows the motion the bubbles and confinement on the surface of the solid material of bubble in conditions of weightlessness.

\section{Conclusions}

The developed model and numerical calculations permitted to determine that under increased surface tension and correspondingly decreased cohesive contact angle in microgravity $\left(\mathrm{g}=196 \mathrm{~cm} / \mathrm{sec}^{2}\right)$ conditions, the gas bubble (drop) is "raised" on the solid surface in the fluid and its free common surface energy increased.

In this case, the gas bubble (drop) is more tore from solid surface because of decrease of the resultant force, which retains bubble on the surface of solid material, and its increased free energy is transformed to the increased kinetic energy of bubble jumping from top to the fluid bottom.

Under decreased surface tension and correspondingly increased cohesive contact angle at microgravity $\left(\mathrm{g}=196 \mathrm{~cm} / \mathrm{sec}^{2}\right)$, the gas bubble (drop) is "laid" on the solid surface in the fluid, the resultant force, which retains bubble on the surface of solid material, is increased and its free common surface energy is decreased. In this case, the gas bubble (drop) is fixed on the solid surface and its free common surface energy is expensed on the bubble (drop) oscillations at the solid surface.

The theoretical conclusions and numerical calculations of the developed computational model have been proved on the conducted parabolic aircraft's tests and permitted to realize the control of the behavior of gas bubble in a fluid using surface tension and cohesive angle change under decreased gravity.

\section{References}

1. Bashforth $F$, Adams $J$ (1883) An attempt to test the theories of capillary action by comparing the theoretical and measured forms of drops of fluid. With an explanation of the method of integration employed in constucting the tables which give the theoretical forms of such drops. Cambridge.

2. Padday JF (1971) The profiles of axially symmetric menisci. Phys Trans Roy Soc 269: 1197.

3. Shoikhedbrod M.P (1981) Gas phase behavior in a fluid under reduced gravity conditions. Preprints of the Reports at the Republican scientific conference of Tajik SSR's young scientists and specialists, Dushanbe, USSR, 41.

4. Shoikhedbrod MP (2017) The gas bubbles behavior in variable gravity. Lambert Academic Publishing, Toronto.

5. Gagarin $Y$ (1983) Protocols of Reduced Gravity Research Aircraft in Parabolic Flight Tests N1/208009/17, N11/208209/15," The Yuri Gagarin Cosmonauts Training Center, Moscow Region. 